\title{
ON COMPLEXITY OF MODEL-CHECKING FOR THE TQL LOGIC
}

\author{
Iovka Boneva, Jean-Marc Talbot \\ Laboratoire d'Informatique Fondamentale de Lille, France \\ INRIA team Mostrare
}

\begin{abstract}
In this paper we study the complexity of the model-checking problem for the tree logic introduced as the basis for the query language TQL [Cardelli and Ghelli, 2001]. We define two distinct fragments of this logic: $T L$ containing only spatial connectives and $T L^{\exists}$ containing spatial connectives and quantification. We show that the combined complexity of $T L$ is PSPACE-hard. We also study data complexity of model-checking and show that it is linear for $T L$, hard for all levels of the polynomial hierarchy for $T L^{\exists}$ and PSPACE-hard for the full logic. Finally we devise a polynomial space model-checking algorithm showing this way that the model-checking problem for the TQL logic is PSPACE-complete.
\end{abstract}

\section{Introduction}

The development of the WEB made semi-structured data prominent. Semistructured data can be represented by various models such as graphs [Abiteboul et al., 2000], unranked-ordered trees [Neven, 2002] (as for XML documents) or unranked-unordered trees. In [Cardelli, 2000], Cardelli noticed the similarity between this model of unranked-unordered trees and processes from the ambient calculus [Cardelli and Gordon, 2000b]. He also suggested that the ambient logic [Cardelli and Gordon, 2000a] could be a formalism for querying such data representation. Following those ideas, Cardelli and Ghelli proposed in [Cardelli and Ghelli, 2001] TQL (Tree Query Language). The main features of TQL are a semi-structured data representation based on (static) ambient processes and a query formalism based on a tree logic which is an extension of the spatial fragment of the ambient logic. The TQL logic is a very expressive logic which contains Boolean operations, spatial connectives, quantification over labels and trees and a fixed point operator.

The model-checking problem is to decide for a tree and a formula, whether the tree satisfies the formula. In the context of databases, this problem (also called Boolean query) can be used to test whether a database satisfies some integrity constraints. We focus in this paper on the model-checking problem 
for the TQL logic and two natural fragments of it: we investigate in particular the combined and data complexity for these fragments; we recall that the combined complexity of model-checking is the complexity of the model-checking problem when both the tree and the formula are inputs of the problem. The data complexity is the complexity considering only the tree as input of the problem.

One of our main results is that combined complexity for model-checking for the tree logic is PSPACE-complete. To prove complexity upper bound, we design a polynomial space model-checking algorithm inspired from the local model-checking of the $\mu$-calculus [Stirling and Walker, 1991]. Combined complexity of model-checking for the tree logic is already known to be PSPACE-hard [Charatonik et al., 2001]. For the proof of this latter, the authors use quantification over labels. We show here that PSPACE-hardness of model-checking is obtained even for the smallest spatial fragment of the tree logic, that is, the fragment containing only spatial connectives and Boolean operators (we call this fragment $T L$ ). This result is surprising as it shows that quantification and fixed point operator, although adding expressive power to the logic, do not make model-checking harder.

Our second contribution concerns the data complexity of model-checking: we show that the data complexity is linear for the $T L$ fragment and PSPACEhard for the full logic. We obtain also an intermediate result: the data complexity of the fragment obtained by removing fixed point operators from the full logic is hard for any level of the polynomial hierarchy.

The paper is organized as follows: in Sect. 2, we introduce information trees (a representation as nested multisets for edge-labeled, unranked and unordered trees). We give also the syntax and the semantics of the tree logic proposed by Cardelli and Ghelli and define two fragments of this logic. Sect. 3 is devoted to the study of complexity lower bounds of the model-checking problem for the tree logic and its fragments. Finally, we present in Sect. 4 a polynomial space model-checking algorithm for the tree logic.

\section{Definitions}

\subsection{The Tree Model}

We consider edge-labeled, unranked and unordered trees represented, following [Cardelli and Ghelli, 2001], as nested multisets. This is a quite natural interpretation when dealing with unordered unranked trees.

We denote $\{\{\}$ the empty multiset and $\uplus$ the multiset union. We consider a countable set of labels $\Lambda$ and we define the set $\mathcal{D}$ of information trees ${ }^{1}$ as the

\footnotetext{
${ }^{1}$ In [Cardelli and Ghelli, 2001], the authors considered additionally the notion of info-terms, which correspond to ambient processes. They also define a denotation for info-terms in terms of information trees. Here we omit this notion of info-terms to speak directly about information trees.
} 
least one satisfying: (i) the empty multiset $\{\{\}$ is in $\mathcal{D}$, (ii) if $m$ is a label from $\Lambda$ and $A$ belongs to $\mathcal{D}$ then $\{m[A]\}$ belongs to $\mathcal{D}$ and (iii) if A, $A^{\prime}$ belong to $\mathcal{D}$ then $A \uplus A^{\prime}$ belongs to $\mathcal{D}$.

For any tree $A$, we denote $f n(A)$ the set of labels appearing in $A$.

We define the component relation as a partial ordering relation over information trees recursively defined as follows: a tree $A$ is a component of a tree $A^{\prime}$ (denoted $A \sqsubseteq A^{\prime}$ ) if either $A$ is a subset of $A^{\prime}$ or, for some element $m[B]$ in $A^{\prime}, A$ is a component of $B$. The component relation on information trees differs from the usual subtree relation: considering the tree structure of some information tree $A$, a subtree can be identified by a single node in $A$ whereas a component of $A$ is characterized by a set of sibling nodes. We use a different term to avoid counter-intuitions. Note also that the number of subtrees for some tree $A$ is linear in the size of $A$ whereas the number of components of $A$ may be exponential in the size of this tree.

\subsection{The Tree Logics $T L_{\nu}^{\exists}, T L^{\exists}$ and $T L$}

This section presents the tree logic defined in [Cardelli and Ghelli, 2001], that we denote $T L_{\nu}^{\exists}$, as well as its two fragments $T L^{\exists}$ and $T L$.

Syntax. We assume a countable set $\mathcal{L}$ of label variables ranging over by $x, y$, a countable set $\mathcal{T}$ of tree variables ranging over by $X, Y$ and a countable set $\boldsymbol{R}$ of recursion variables ranging over by $\boldsymbol{\xi}$. Let $\boldsymbol{\eta}$ be a label constant or a label variable. Formulas of the logic $T L_{\nu}^{\exists}$ are defined recursively as :

- 0 (empty tree), $\mathrm{T}$ (true), $X$ (tree variable), $\boldsymbol{\xi}$ (recursion variable) and $\eta=\eta^{\prime}$ (label equality test) are formulas from $T L_{\nu}^{\exists}$;

- if $\varphi, \psi$ are formulas from $T L_{\nu}^{\exists}$ then $\eta[\varphi]$ (location), $\varphi \mid \psi$ (composition), $\neg \varphi$ (negation), $\varphi \vee \psi$ (disjunction), $\exists x . \varphi$ (quantification over label variables) $\exists X . \varphi$ (quantification over tree variables) and $\boldsymbol{\nu} \boldsymbol{\xi} . \boldsymbol{\varphi}$ (greatest fixed point) are formulas from $T L_{\nu}^{\exists}$.

To guarantee the existence of the greatest fixed point $\nu \xi . \varphi$, we require that any occurrence of the recursion variable $\xi$ in the formula $\varphi$ is in the scope of an even number of negations.

The derived operators $\varphi \wedge \psi, \forall x . \varphi, \forall X . \varphi$ and $\mu \xi . \varphi$ are defined as usual.

Binding operators are $\exists x$ for label variables, $\exists X$ for tree variables and $\boldsymbol{\nu} \xi$ for recursion variables. Let $f v(\varphi)$ be the set of free variables of the formula $\varphi$. We say that a formula is closed if it does not contains free variables.

We denote $f n(\varphi)$ the set of labels occurring in the formula $\varphi$. >From now on, we assume that in formulas free variables are disjoint from bounded variables and moreover, that two distinct occurrences of binders bind different variables. 
Semantics and Satisfiability. We consider valuations $\rho$ and $\delta$ defined over a finite subset of respectively $\mathcal{L} \cup \mathcal{T}$ and $\mathcal{R}$. The valuation $\rho$ maps label variables to labels and tree variables to trees whereas the valuation $\delta$ maps recursion variables to subsets of $\mathcal{D}$. For $\rho$ (resp. $\delta$ ), we denote $\operatorname{dom}(\rho)$ (resp. $\operatorname{dom}(\delta))$ its domain.

The set of free label- and tree-variables of a formula $\varphi$ under a valuation $\rho$ denoted $f v(\varphi, \rho)$ is defined as $(f v(\varphi) \cap(\mathcal{L} \cup \mathcal{T})) \backslash \operatorname{dom}(\rho)$. Respectively, the set of free recursion variables of a formula $\varphi$ under a valuation $\delta$ denoted $f v(\varphi, \delta)$ is defined as $(f v(\varphi) \cap \mathcal{R}) \backslash \operatorname{dom}(\delta)$. We say that a formula $\varphi$ is closed under a valuation $\rho$, or simply $\rho$-closed (resp. under a valuation $\delta$, or $\delta$-closed) if $f v(\varphi, \rho)=\varnothing$ (resp. $f v(\varphi, \delta)=\varnothing)$.

The interpretation of the formula $\varphi$ is given by a mapping $\llbracket \varphi \rrbracket_{\rho, \delta}$ which associates with $\varphi$ a subset of $\mathcal{D}$. This mapping is parametrized by two valuations $\rho$ and $\delta$ such that $\varphi$ is both $\rho$ - and $\delta$-closed. We assume that $\rho$ is extended on labels from $\Lambda$ and maps any label to itself; thus, we can write $\rho(\eta)$ for constant labels $\eta$. The valuation $\rho[x \mapsto n]$ is identical to $\rho$ except for $x$ which is mapped to $n$; the valuations $\rho[X \mapsto A]$ and $\delta[\xi \mapsto S]$ are defined in a similar way.

Interpretation of formulas

\begin{tabular}{|c|c|c|}
\hline $\begin{array}{l}\llbracket 0 \rrbracket_{\rho, \delta} \\
\llbracket \top T \rrbracket_{\rho, \delta} \\
\llbracket \neg \varphi \rrbracket_{\rho, \delta} \\
\llbracket \exists x . \varphi \rrbracket_{\rho, \delta} \\
\llbracket X \rrbracket_{\rho, \delta}\end{array}$ & $\begin{array}{l}=\{\{\{\}\} \\
=\mathcal{D} \\
=\mathcal{D} \backslash \llbracket \varphi \rrbracket_{\rho, \delta} \\
=\bigcup_{n \in \Lambda} \llbracket \varphi \rrbracket_{\rho[\rho-n], \delta} \\
=\{\rho(X)\} \\
=\delta(\xi)\end{array}$ & $\begin{array}{l}\llbracket \eta[\varphi] \rrbracket_{\rho, \delta}=\left\{\left\{\{\rho(\eta)[A]\} \mid A \in \llbracket \varphi \rrbracket_{\rho, \delta}\right\}\right. \\
\llbracket \varphi \mid \psi \rrbracket_{\rho, \delta}=\left\{A \uplus B \mid A \in \llbracket \varphi \rrbracket_{\rho, \delta}, B \in \llbracket \psi \rrbracket_{\rho, \delta}\right\} \\
\llbracket \varphi \vee \psi \rrbracket_{\rho, \delta}=\llbracket \varphi \rrbracket_{\rho, \delta} \cup \llbracket \psi \rrbracket_{\rho, \delta} \\
\llbracket \eta=\eta^{\prime} \rrbracket_{\rho, \delta}=\mathcal{D} \text { if } \rho(\eta)=\rho\left(\eta^{\prime}\right), \varnothing \text { otherwise } \\
\llbracket \exists X . \varphi \rrbracket_{\rho, \delta}=\bigcup_{A \in \mathcal{A}} \llbracket \llbracket \rrbracket_{\rho[X \rightarrow A], \delta} \\
\llbracket \nu \xi . \varphi \rrbracket_{\rho, \delta}=\bigcup\left\{S \subseteq \mathcal{D} \mid S \subseteq \llbracket \varphi \rrbracket_{\rho, \delta[\xi-S]}\right\}\end{array}$ \\
\hline
\end{tabular}

Definition 1 (SATISFIABILITY) For any tree A, any formula $\varphi$, and any valuations $\rho, \delta$ such that $\varphi$ is $\rho$ - and $\delta$-closed, the tree $A$ satisfies the formula $\varphi$ under the valuations $\rho$ and $\delta$, written $A \models_{\rho, \delta} \varphi$, if $A$ is in the set $\llbracket \varphi \rrbracket_{\rho, \delta}$.

In the notations $A \models_{\rho, \delta} \varphi$ and $\llbracket \varphi \rrbracket_{\rho, \delta}$ we sometimes omit $\rho$ (resp. $\delta$ ) whenever the formula $\varphi$ contains no free label- and tree-variables (resp. no free recursion variables).

Fragments of the Logic. In the following of this paper, we will consider two fragments of the tree logic $T L_{\nu}^{\exists}$; the first fragment, denoted $T L$, is the smallest spatial logic included in $T L_{\nu}^{\exists}$ as it is defined only with Boolean operators, the empty tree, composition and location. The second fragment, denoted $T L^{\exists}$, is obtained by removing fixed point operator from the logic $T L_{\nu}^{\exists}$. It is easy to see that $T L \subseteq T L^{\exists} \subseteq T L_{\nu}^{\exists}$.

\subsection{Comparison with Other Logics}

We show in this section that the monadic second-order logic over trees (MSO) is more expressive than the $T L$ fragment but that the logic $T L^{\exists}$ can express properties that can not be expressed in MSO. 
We consider $\Lambda^{\prime} \subseteq \Lambda$ a finite set of labels. Let $\boldsymbol{\tau}$ be the signature \{label $\boldsymbol{n}_{\boldsymbol{n}}$ | $\left.n \in \Lambda^{\prime}\right\} \cup\{<\}$ where the label $\left.\right|_{n}$ 's are unary predicates and $<$ is a binary predicate. An information tree $A$ (with $f n(A) \subseteq \Lambda^{\prime}$ ) can be represented by a finite $\tau$-structure $T^{A}=\left\langle E^{A},\left\{\right.\right.$ label $\left.\left.{ }_{n}^{A} \mid n \in \Lambda^{\prime}\right\},<^{A}\right\}$ where $E^{A}$ is a finite set of edges, label ${ }_{n}^{A}$ associates the label $n$ with edges, and $<^{A}$ is a binary relation over edges such that $u<u^{\prime}$ holds iff the destination of the edge $u$ and the source of the edge $u^{\prime}$ coincide.

We consider MSO over the signature $\tau$. Edge variables range over $\boldsymbol{u}, \boldsymbol{v}$ and set variables range over $U, V$. For any $T L$ formula $\varphi$, we define an MSO formula $R_{\varphi}(U)$ parametrized by the set of edges $U$, such that for any information tree A, $A \models \varphi$ iff $T^{A} \vDash \forall U .\left((\forall u . u \in U) \rightarrow R_{\varphi}(U)\right)$.

\section{Encoding of $T L$ in MSO}

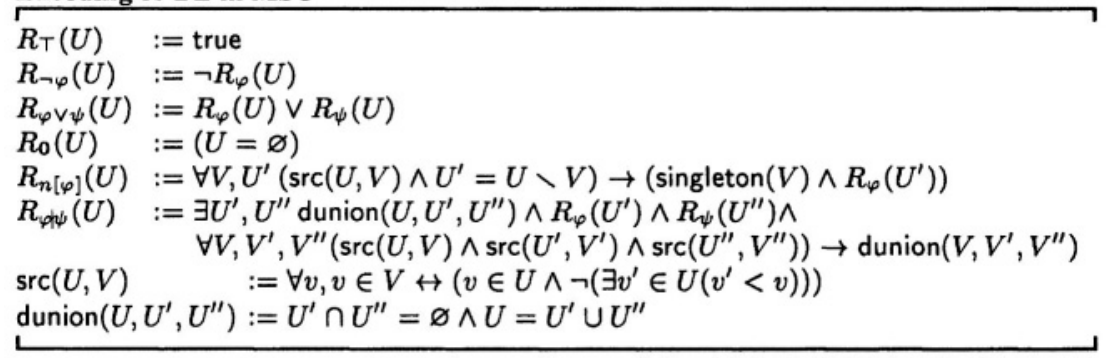

Now, consider the $T L^{\exists}$ formula $\exists X(a[X] \mid b[X])$ : it expresses that the root of the tree has two direct subtrees (reachable by $\boldsymbol{a}$ and $\boldsymbol{b}$ ) and that these subtrees are isomorphic. It is well-know that this property can not be expressed in MSO.

Some other logics express properties of unranked and unordered trees: for instance, the monadic second order logic with counting (CMSO) [Courcelle, 1990b], the Presburger monadic second order logic (PMSO) [Seidl et al., 2003], the sheaves logic [Dal-Zilio and Lugiez, 2003]. We strongly believe that the tree logic we consider in this paper is strictly more powerful than those logics.

\section{Complexity of Model-Checking}

The model-checking problem is given an information tree $A$, a valuation $\rho^{2}$ and a $\rho$-closed formula $\varphi$ without free recursion variable, decide whether $A \models \rho$ $\varphi$. We study in this section lower bounds of the combined complexity and the data complexity of model-checking for the tree logic $T L_{\nu}^{\Im}$ and its fragments. We establish that combined complexity is PSPACE-hard for the less expressive fragment $T L$ and that data-complexity is linear for $T L$, hard for any level of the polynomial hierarchy for $T L^{\exists}$ and PSPACE-hard for the full logic $T L_{\nu}^{\exists}$.

For PSPACE-hardness (resp. hardness for levels in the polynomial hierarchy) proof, we use reductions of the validity problem of closed quantified

\footnotetext{
${ }^{2}$ The valuation $\rho$ is considered to be empty for the logic $T L$.
} 
Boolean formulas (QBF) (resp. of closed QBF with fixed quantifier alternation depth) to the model-checking problem.

\subsection{Quantified Boolean Formulas}

A quantified Boolean formula $(\mathrm{QBF})$ is a formula $Q_{1} v_{1} \ldots Q_{n} v_{n} . G_{\text {where }}$ each $Q_{\boldsymbol{i}}$ is a quantifier among $\exists$ and $\forall$ and $G$ is a prepositional logic formula built on the set of variables $\left\{v_{1}, \ldots, v_{n}\right\}$. We can assume without loss of generality that $G$ is in disjunctive normal form and follows the syntax:

$$
G::=\theta_{1} \vee \ldots \vee \theta_{k} \quad \theta::=l_{1} \wedge \ldots \wedge l_{r} \quad l::=v_{i} \mid \overline{v_{i}}, i \in\{1, \ldots, n\}
$$

It is well known that validity of closed QBF is a PSPACE-complete problem [Stockmeyer, 1976].

The quantifier alternation depth $\operatorname{depth}(\mathcal{G})$ of a QBF $\mathcal{G}$ is defined as: (i) for a quantifier-free formula $\mathcal{G}$, $\operatorname{depth}(\mathcal{G})=0$ and $\operatorname{depth}(Q x . \mathcal{G})=1$ (for $Q \in\{\exists, \forall\})$, (ii) $\operatorname{depth}\left(Q x . Q^{\prime} x^{\prime} \cdot \mathcal{G}\right)=\operatorname{depth}\left(Q^{\prime} x^{\prime} \cdot \mathcal{G}\right)$ if $Q=Q^{\prime}$ and (iii) $\operatorname{depth}\left(Q x \cdot Q^{\prime} x^{\prime} \cdot \mathcal{G}\right)=1+\operatorname{depth}\left(Q^{\prime} x^{\prime} \cdot \mathcal{G}\right)$ if $Q \neq Q^{\prime}$

The validity problem of a closed QBF of alternation depth $n$ whose outermost quantifier is $\forall$ (resp. $\exists$ ) is complete for the universal (resp. existential) $n^{\text {th }}$ level of the polynomial hierarchy $P H$, that is $\Pi_{n}^{P}$-complete (resp. $\Sigma_{n}^{P}$ complete) [Stockmeyer, 1976].

\subsection{Combined Complexity}

The combined complexity of the model-checking problem $A \models \rho \varphi$ is the complexity of the problem considering $A, \varphi$ and $\rho$ as inputs of the problem.

It has already been shown in [Charatonik et al., 2001] that the combined complexity of $T L^{\exists}$ is PSPACE-hard by encoding the validity problem of QBF into the model-checking of $T L^{\exists}$. This encoding uses only Boolean connectives, label comparison and existential quantification over labels. We show here that the combined complexity is PSPACE-hard even for the less expressive logic $T L$.

With any QBF $\mathcal{F}$, we associate the $T L$ formula $(\mathcal{F})$ defined as:

\section{Encoding of QBF into TL}

\begin{tabular}{|c|c|c|c|}
\hline $\begin{array}{l}\left(v_{i}\right) \\
\left(\exists v_{i} . \mathcal{F}\right) \\
\left(l_{1} \wedge \ldots \wedge l_{k}\right)\end{array}$ & $\begin{array}{l}=v_{i}[t[0]] \\
=v_{i}[T] \mid(\mathcal{F D}) \\
=\left(l_{1} D\left|T \wedge \ldots \wedge\left(l_{k}\right)\right| T\right.\end{array}$ & $\begin{array}{l}\left(\overline{v_{i}}\right) \\
\left.\partial \forall v_{i} \mathcal{F}\right) \\
\left(\theta_{1} \vee \ldots \vee \theta_{k}\right\rangle\end{array}$ & $\begin{array}{l}=v_{i}[f[0]] \\
=\neg\left(v_{i}[T] \mid \neg(\mathcal{F})\right) \\
=\left(\theta_{1}\right) \vee \ldots \vee\left(\theta_{k}\right)\end{array}$ \\
\hline
\end{tabular}

where $t, f$ and the $v_{i}$ are labels from $\Lambda$.

Let $T$ and $F$ be respectively the information trees $\{\{t[\{\}\}]\}\}$ and $\{\{f[\{\}\}]\}$. We consider in the sequel the $n$-variables QBF $\mathcal{G}=Q_{1} v_{1} \ldots Q_{n} v_{n} . G$. Let $P_{\mathfrak{g}}$ be the constant information tree (note that the construction of $P_{\mathcal{G}}$ is poly- 
nomial time)

$$
P_{\mathcal{G}}=\left\{\left\{v_{1}[T], v_{1}[F], v_{2}[T], v_{2}[F], \ldots, v_{n}[T], v_{n}[F]\right\}\right.
$$

\section{LEMMA 2 For any closed quantified Boolean formula $\mathcal{G}, P_{\mathcal{G}}=(\mathcal{G} \mid$ iff $\mathcal{G}$ is} valid.

We don't give here the full proof of the lemma, but just an intuitive idea about the encoding. Remark first that the tree $\boldsymbol{P}_{\mathcal{C}}$ is the multiset of the elements $v_{i}[T]$ and $v_{i}[F]$ for all the variables $v_{i}$ occurring in $G$. On the other hand, a valuation for the variables $\boldsymbol{v}_{\boldsymbol{i}}$ in $G$ can be viewed as a component $\boldsymbol{P}^{\prime}$ of $P_{\mathcal{g}}$ in which each of the $v_{i}$ occurs only once either as $v_{i}[T]$ or as $v_{i}[F]$, and $T$ and $F$ determine the valuation of the variable $v_{i}$. The tree $P^{\prime}$ can be constructed from $P_{\mathfrak{g}}$ by $n$ successive eliminations of one of the elements $v_{i}[T]$ and $v_{i}[F]$ for each $i$ in $\{1, \ldots, n\}$. Hence, the model-checking problem $P_{\mathcal{O}} \models(\mathcal{G})$ can be viewed as the construction of a valuation for the set of variables $\left\{v_{1}, \ldots, v_{n}\right\}$ by elimination of elements in $\boldsymbol{P}_{\boldsymbol{g}}$, followed by the verification that this valuation renders the formula $G$ true. The elimination of the element $\boldsymbol{v}_{\boldsymbol{i}}[\boldsymbol{Z}]$ for $\boldsymbol{Z} \in\{\boldsymbol{T}, \boldsymbol{F}\}$ is done considering $\boldsymbol{P}_{\mathcal{C}}$ as the union $\left\{v_{i}[Z]\right\} \uplus P^{\prime}$. Thus, model-checking $\left.\left|\exists v_{i} . \mathcal{F}\right\rangle=v_{i}[T] \mid(\mathcal{F}\rangle\right)$ leads us to eliminate non-deterministically one of the elements $\left(v_{i}[T]\right.$ or $\left.v_{i}[F]\right)$ while modelchecking $\left(\forall v_{i}, \mathcal{F}\right)=\neg\left(v_{i}[T] \mid \neg(\mathcal{F})\right)$ leads us to consider both cases in which $v_{i}[T]$ and $v_{i}[F]$ are eliminated.

THEOREM 3 The combined complexity of model-checking the tree logic TL is PSPACE-hard.

\subsection{Data Complexity}

The data complexity of the model-checking problem $A \models_{\rho} \varphi$ is the complexity of the problem for some fixed closed formula $\boldsymbol{\varphi}$ and valuation $\boldsymbol{\rho}$. That is, only the tree $A$ is considered as input for the problem. We show in the following that the data complexity of model-checking is PSPACE-hard for $T L_{\nu}^{\exists}$, hard for any level of the polynomial hierarchy for $T L^{\exists}$ and linear for $T L$.

Let us first introduce some objects which are in common to the next two encodings. Let $\boldsymbol{G}=\boldsymbol{\theta}_{\mathbf{1}} \vee \ldots \vee \boldsymbol{\theta}_{\boldsymbol{k}}$ be a Boolean formula in disjunctive normal form constructed over the set of variables $\bigcup_{i \in I}\left\{v_{i}\right\}$ for some finite set $I$. Moreover, we require that the $\boldsymbol{\theta}_{j}$ are not trivially false, that is, for any $\boldsymbol{j}$ in $1 . . k$, there is no $\boldsymbol{i}$ in $I$ such that both $\boldsymbol{v}_{\boldsymbol{i}}$ and $\overline{\boldsymbol{v}}_{\boldsymbol{i}}$ occur in $\boldsymbol{\theta}_{\boldsymbol{j}}$.

For any $i$ in $I$, we define the sets $C_{i}^{\text {true }} \subseteq\{1, \ldots, k\}$ and $C_{i}^{\text {false }} \subseteq\{1, \ldots, k\}$ as: $j$ is in $C_{i}^{\text {true }}$ if the variable $v_{i}$ does not occur negatively in $\theta_{j}$ and $j$ is in $C_{i}^{\text {false }}$ if the variable $v_{i}$ does not occur positively in $\theta_{j}$. Assuming that $\mathrm{c}_{j}$, for $j$ in $1 . . k$, is a label from $\Lambda$, we define the information trees $C_{i}^{t}=$ $\biguplus_{j \in C_{i}^{\text {true }}}\left\{\left\{c_{j}[\{\}\}\right]\right\}$ and $C_{i}^{f}=\biguplus_{j \in C_{i}^{\text {false }}}\left\{\left\{c_{j}[\{\}\}\right]\right\}$. 
A valuation $\gamma$ for $G$ is a mapping from $I$ to \{true, false \}. It is easy to see that the formula $G$ is valid for some valuation $\gamma$ iff there exists some $j \in 1$...k such that $j$ is in $C_{i}^{\gamma(i)}$ for any $i$ in $I$.

$\boldsymbol{T} \boldsymbol{L}_{\boldsymbol{\nu}}^{\exists}$. We show here that the data complexity of the model-checking problem for the tree logic $T L_{\nu}^{\exists}$ is PSPACE-hard, that is, there exists a fixed $T L_{\nu}^{\exists}$ formula $\Phi$ such that the model-checking problem "given the information tree $A$, does $A=\Phi$ hold" is PSPACE-hard.

The PSPACE-hardness proof is done using a reduction of the validity problem of a closed QBF into the model-checking problem for $T L_{\nu}^{\exists}$. With any QBF $\mathcal{G}$, we associate the information tree $A_{\mathcal{G}}$ such that $A_{\mathcal{G}} \models \Phi$ iff $\mathcal{G}$ is valid.

Consider the $n$-variables QBF $\mathcal{G}=Q_{1} v_{1} \ldots Q_{n} v_{n} . G$. Let $v_{i}$ for $i \in 1 . . n$, $c_{j}$ for $j \in 1 . . k, v_{0}, q_{\forall}, q_{\exists}, \mathrm{tt}$, ff, pred, quant and sat be labels. Let

$$
\left.A_{\mathcal{G}}=\left\{\left\{v_{0}[\{\}\}\right], V_{1}^{\text {true }}, V_{1}^{\text {false }}, \ldots, V_{n}^{\text {true }}, V_{n}^{\text {false }}\right\}\right\}
$$

where, for any $i \in 1$...n, the trees $V_{i}^{\text {true }}$ and $V_{i}^{\text {false }}$ are

$$
\begin{aligned}
& V_{i}^{\text {true }}=v_{i}\left[\left\{\operatorname{tt}\left[\{\{\}], \text { quant }\left[\left\{q_{Q_{i}}[\{\{\}]\}\right\}\right], \text { pred }\left[\left\{v_{i-1}[\{\{\}]\}\right\}\right], \text { sat }\left[C_{i}^{t}\right]\right\}\right\}\right] \\
& V_{i}^{\text {false }}=v_{i}\left[\{f f[\{\}\}], \text { quant }\left[\left\{\left\{q_{Q_{i}}[\{\{\}]\}\right], \text { pred }\left[\left\{v_{i-1}[\{\{\}]\}\right], \text { sat }\left[C_{i}^{f}\right]\right\}\right\}\right]\right.
\end{aligned}
$$

Intuitively, for any variable $v_{i}$ occurring in the $\mathrm{QBF} \mathcal{G}$, the information tree $A_{\mathcal{G}}$ encodes the kind of quantification of $v_{i}$ (in the component quant $\left.\left[\left\{q_{Q_{i}}[\{\{\}]\}\right\}\right]\right)$ and the rank of the variable (giving the previous variable in the component pred). The information trees $C_{i}^{t}$ and $C_{i}^{f}$ encode the formula $\mathrm{G}$.

The $T L_{\nu}^{\exists}$ formula $\Phi$ is defined as

$$
\Phi=\mu \xi .(\neg I \text { Valuation } \wedge \text { Construct Val }) \vee(\text { Is Valuation } \wedge \text { Valid })
$$

The mechanism used here is similar to the one used to prove combined complexity lower bound of $T L$ (Sect. 3.2). Intuitively, the formula $\Phi$ constructs all possible valuations for the variables $v_{i}$ regarding their quantification in $\mathcal{G}$ and then verifies, for each valuation, whether it valuates the formula $G$ to true.

To any valuation $\gamma$ corresponds exactly a component of $A_{\mathscr{g}}$ denoted $A_{\mathcal{G}}\{\gamma\}$ and defined as $\left\{v_{0}[\{\}], V_{1}^{\gamma(1)}, V_{2}^{\gamma(2)}, \ldots, V_{n}^{\gamma(n)}\right\}$. Note that in $A_{\mathcal{O}}\{\gamma\}$, any of the $v_{i}$ occurs only once. Therefore, the formula IsValuation verifying whether a component of $A_{\mathcal{G}}$ corresponds to a valuation is defined as:

$$
\text { Is Valuation }=\neg\left(\exists x_{\mathrm{v}} \cdot x_{\mathrm{v}}[T]\left|x_{\mathrm{v}}[\top]\right| \top\right)
$$

Now, a valuation can be constructed from $A_{\mathcal{G}}$ by $n$ successive eliminations of one of $V_{i}^{\text {true }}$ or $V_{i}^{\text {false }}$, for any $i \in\{1, \ldots, n\}$. All possible valuations of the variables $v_{i}$, according to the quantifications of these variables, are enumerated by the $T L_{\nu}^{\exists}$ subformula Construct Val which is the recursive part of $\Phi$. In the 
following, we let $\operatorname{Forall}\left(x_{\mathrm{v}}\right)=x_{\mathrm{v}}\left[\right.$ quant $\left.\left._{\left[q_{v}\right.}[\mathbf{0}]\right] \mid \mathrm{T}\right] \mid \mathrm{T}$ and $\operatorname{Exists}\left(x_{\mathrm{v}}\right)=$ $x_{\vee}\left[\right.$ quant $\left.\left[q_{\exists}[0]\right] \mid \mathrm{T}\right] \mid \mathrm{T}$.

$$
\begin{aligned}
& \text { Construct Val }=\exists x_{\mathrm{v}} . \text { FirstNon Val }\left(x_{\mathrm{v}}\right) \wedge\left(\begin{array}{c}
\text { Forall }\left(x_{\mathrm{v}}\right) \wedge \neg\left(x_{\mathrm{v}}[T] \mid \neg \xi\right) \\
\vee \\
\operatorname{Exists}\left(x_{\mathrm{v}}\right) \wedge x_{\mathrm{v}}[T] \mid \xi
\end{array}\right) \\
& \text { FirstNonVal }\left(x_{v}\right)=\exists x_{v}^{\prime} \cdot\left(\begin{array}{c}
\neg\left(x_{v}^{\prime}[T]\left|x_{v}^{\prime}[T]\right| T\right) \\
\wedge \\
x_{v}[T]\left|x_{v}[T]\right| T \\
\wedge \\
x_{v}\left[\operatorname{pred}\left[x_{v}^{\prime}[T]\right] \mid T\right] \mid T
\end{array}\right)
\end{aligned}
$$

Finally, according to the definitions of the sets $C_{i}^{\text {true }}$ and $C_{i}^{\text {false }}$, to verify the validity of $G$ under the valuation $\boldsymbol{\gamma}$ amounts to test the existence of some $j \in$ $\{1, \ldots, k\}$ such that $\left.c_{j}[\{\}\}\right]$ is a component of $C_{i}^{\gamma(i)}$ for any $i$. This is done by the formula Valid which is the base case for $\Phi$.

$$
\begin{aligned}
\text { Valid } & =v_{0}[T] \mid \exists x_{c} \text {.Everywhere }\left(x_{c}\right) \\
\text { Everywhere }\left(x_{\mathrm{c}}\right) & =\forall x_{\mathrm{v}} \cdot\left(x_{\mathrm{v}}[\mathrm{T}]\left|\mathrm{T} \rightarrow x_{\mathrm{v}}\left[\operatorname{sat}\left[x_{\mathrm{c}}[0] \mid \mathrm{T}\right] \mid \mathrm{T}\right]\right| \mathrm{T}\right)
\end{aligned}
$$

Lemma 4 embodies the correctness of the reduction and implies Theorem 5 simply by noticing that the size of the tree $A_{\mathcal{g}}$ is polynomial in the size of the QBF $\mathcal{G}$.

Lemma 4 The closed quantified Boolean formula $\mathcal{G}$ is valid iff $\boldsymbol{A}_{\mathfrak{g}} \models \boldsymbol{\Phi}$.

THEOREM 5 The data complexity of model-checking the tree logic $T L_{\nu}^{\exists}$ is PSPACE-hard.

$\boldsymbol{T} \boldsymbol{L}^{\exists}$. We show here that the data complexity of the model-checking problem for the tree logic $T L^{\exists}$ is hard for any level of the polynomial hierarchy $P H$. That is, we show that for any universal (resp. existential) level of $P H \Pi_{n}^{P}$ (resp. $\left.\Sigma_{n}^{P}\right)$, there exists a fixed formula $\boldsymbol{\Phi}_{n}^{\forall}$ (resp. $\boldsymbol{\Phi}_{n}^{\exists}$ ) such that the model-checking problem "given the information tree $A$, does $A \models \Phi_{n}^{\forall}$ (resp. $A=\Phi_{n}^{\exists}$ ) hold" is $\Pi_{n}^{P}$-hard (resp. $\Sigma_{n}^{P}$-hard).

To prove $\Pi_{n}^{P}$-hardness (resp. $\Sigma_{n}^{P}$-hardness), we present a reduction of the validity problem of closed QBF with alternation depth $n$ and universal (resp. existential) outermost quantifier into the model-checking problem for $T L^{\exists}$ with some fixed formula $\boldsymbol{\Phi}_{n}^{\forall}$ (resp. $\Phi_{n}^{\exists}$ ). More precisely, with any closed QBF $\mathcal{G}$ of alternation depth $n$ we associate the information tree $A_{\mathcal{G}}$ and we show that $A_{\mathcal{G}} \models \Phi_{n}^{\forall}$ (resp. $A_{\mathcal{G}} \vDash \Phi_{n}^{\exists}$ ) iff the outermost quantifier of $\mathcal{G}$ is $\forall$ (resp. $\exists$ ) and $\mathcal{G}$ is valid.

Consider the closed QBF with alternation depth $n\left(Q_{i} \neq Q_{i+1}\right.$ for $i$ in $1 . . n-1)$.

$$
\mathcal{G}=Q_{1} v_{(1,1)} \ldots v_{\left(1, n_{1}\right)} \cdot Q_{2} v_{(2,1)} \ldots v_{\left(2, n_{2}\right)} \ldots Q_{n} v_{(n, 1)} \ldots v_{\left(n, n_{n}\right)} \cdot G
$$


Let $\mathbf{v}_{(h, i)}$ for any $h \in 1 . . n$ and any $i \in 1 . . n_{h}, \mathrm{c}_{j}$ for any $j \in 1 . . k$, quant, sat and $\mathbf{q}_{i}$ for any $i \in 1$..n be labels from $\Lambda$. The information tree $A_{\mathcal{G}}$ is:

$\left.A_{\mathcal{G}}=\left\{V_{(1,1)}^{\text {true }}, V_{(1,1)}^{\text {false }}, \ldots, V_{\left(1, n_{1}\right)}^{\text {true }}, V_{\left(1, n_{1}\right)}^{\text {false }}, V_{(2,1)}^{\text {true }}, V_{(2,1)}^{\text {false }}, \ldots, V_{\left(n, n_{n}\right)}^{\text {true }}, V_{\left(n, n_{n}\right)}^{\text {false }}\right\}\right\}$

where for any $h \in 1 . . n$ and for any $i \in 1 . . n_{h}, V_{(h, i)}^{\text {true }}$ and $V_{(h, i)}^{\text {false }}$ are

$$
\begin{aligned}
& V_{(h, i)}^{\text {true }}=\left\{\left\{\mathrm{tt}\left[\{\{\}\}, \text { quant }\left[\left\{\left\{\mathrm{q}_{h}[\{\{\}]\}\right\}\right], \text { sat }\left[C_{(h, i)}^{t}\right]\right\}\right\}\right.\right. \\
& \left.\left.V_{(h, i)}^{\text {false }}=\left\{\{f f[\{\}\}], \text { quant }\left[\left\{q_{h}[\{\}\}\right]\right\}\right], \text { sat }\left[C_{(h, i)}^{f}\right]\right\}\right\}
\end{aligned}
$$

The formulas $\Phi_{n}^{\forall}$ and $\Phi_{n}^{\exists}$ are defined respectively as $\Psi_{n}^{\forall}(1)$ and $\Psi_{n}^{\exists}(1)$ where for any $h \in 1 . . n$, the formulas $\Psi_{n}^{\forall}(h)$ and $\Psi_{n}^{\exists}(h)$ are defined recursively:

$$
\begin{aligned}
& \Psi_{n}^{\forall}(h)=\neg\left(\text { Remove }(h) \mid\left(\text { Is Valuation }(h) \wedge \neg \Psi_{n}^{\exists}(h+1)\right)\right) \\
& \Psi_{n}^{\exists}(h)=\operatorname{Remove}(h) \mid\left(\text { IsValuation }(h) \wedge \Psi_{n}^{\forall}(h+1)\right)
\end{aligned}
$$

and $\Psi_{n}^{\forall}(n+1)=\Phi_{n}^{\exists}(n+1)=$ Valid $^{\prime}$.

The intuition for this encoding is similar to the one used in previous section. The formulas $\boldsymbol{\Phi}_{n}^{\forall}$ and $\boldsymbol{\Phi}_{n}^{\exists}$ construct all possible valuations for the variables of the formula $\mathcal{G}$ according to their quantification and check, for each valuation, whether it valuates $G$ to true. More precisely, for any $h \in 1 . . n$, the formula $\Psi_{n}^{\forall}(h)$ (resp. $\Psi_{n}^{\exists}(h)$ ) constructs all possible valuations of the variables $v_{(h, 1)}, v_{(h, 2)}, \ldots, v_{\left(h, n_{h}\right)}$ according to the universal (resp. existential) quantification and reiterates the same mechanism to $h+1$. Therefore, Is Valuation $(h)$ and $\operatorname{Remove}(h)$ are defined as:

$$
\begin{aligned}
& \text { Is Valuation }(h)=\neg \exists x_{\mathrm{v}}\left(x_{\mathrm{v}}\left[\text { quant }\left[\mathrm{q}_{h}[0]\right] \mid T\right] \mid x_{\mathrm{v}}\left[\text { quant }\left[\mathrm{q}_{h}[0]\right] \mid T\right] \mid T\right) \\
& \text { Remove }(h)=\forall x_{\mathrm{v}} \cdot\left(x_{\mathrm{v}}[\mathrm{T}] \mid T \rightarrow x_{\mathrm{v}}\left[\text { quant }\left[\mathrm{q}_{h}[0]\right] \mid T\right] \mid T\right) \wedge I \text { Valuation }(h)
\end{aligned}
$$

The formulas $\Psi_{n}^{\forall}(n+1)$ and $\Psi_{n}^{\exists}(n+1)$ check validity of $G$ for some valuation, so the formula Valid' is defined as $\exists x_{c}$. Everywhere $\left(x_{c}\right)$.

The correctness of the encoding we presented is given by Lemma 6 and the complexity result in Theorem 7 is an easy consequence of this lemma using the fact that the size of the tree $A_{\mathfrak{g}}$ is polynomial in the size of the $\mathrm{QBF} \mathcal{G}$.

LEMMA 6 Let $\mathcal{G}$ be a closed quantified Boolean formula of alternation depth $n$ whose outermost quantifier is universal (resp. existential). The formula $\mathcal{G}$ is valid iff $A_{\mathcal{g}} \vDash \Phi_{n}^{\vee}$ (resp. $A_{\mathfrak{g}} \vDash \Phi_{\eta}^{\exists}$ ).

THEOREM 7 The data complexity of model-checking the tree logic $T L^{\exists}$ is hard for any level of the polynomial hierarchy.

TL. We show here that the data complexity of the model-checking problem for the tree logic $T L$ is linear time using the encoding of $T L$ formulas into MSO presented in Sect. 2.3. 
Remark first that by [Courcelle, 1990a], the data complexity of modelchecking is linear for MSO interpreted over finite graphs of bounded tree-width which are a generalization of unranked unordered trees. Consider the modelchecking problem $A \models \varphi$ where the formula $\varphi$ is fixed. Let $\tau$ be the signature $\left\{\right.$ label $\left._{n} \mid n \in f n(A) \cup f n(\varphi)\right\} \cup\{<\}$, and let $T^{A}$ be the $\tau$-structure corresponding to $A$. It is easy to see that the construction of $T^{A}$ is linear time in the size of $A$. Moreover, as $\varphi$ is fixed, the construction of $\boldsymbol{R}_{\varphi}(U)$ is constant time.

THEOREM 8 The data complexity of model-checking the tree logic TL is linear time.

\section{A Model-Checking Algorithm}

Model-checking for the tree logic $T L_{\nu}^{\exists}$ is non trivial for two reasons. On one hand, the existential quantification operator quantifies label and tree variables over infinite sets. We show here how to handle this problem. On the other hand, the (greatest) fixed point operator from $T L_{\nu}^{\exists}$ differs from the kind of fixed points used to extend the first-order logic (FO) to LFP. In LFP, fixed points are defined over the lattice of relations on nodes of a tree (or graph) whereas in $T L_{\nu}^{\exists}$, fixed points are defined over the lattice of sets of trees.

We use an adaptation of the local model-checking for the modal $\boldsymbol{\mu}$-calculus given in [Stirling and Walker, 1991] to devise a polynomial space modelchecking algorithm. Following the ideas from [Winskel, 1991], our algorithm will actually perform model-checking for a slight extension of $T L_{\nu}^{\exists}$ : the syntax of greatest fixed point operator is now $\boldsymbol{\nu} \boldsymbol{\xi}(M) . \varphi$ where $M$ is a finite subset of $\mathcal{D}$ and its semantics is given by $\bigcup\left\{S \subseteq \mathcal{D} \mid S \subseteq \llbracket \varphi \rrbracket_{\rho, \delta[\xi \rightarrow S]} \cup M\right\}$.

One can notice that the logic $T L_{\nu}^{\exists}$ corresponds to formulas where $M$ is the empty set for all fixed point operators. We extend the definition of labels occurring in a formula by letting $f n(\nu \xi(M) . \varphi)=f n(\varphi) \cup \bigcup_{A \in M} f n(A)$.

\subsection{Properties of Satisfaction}

The two propositions here after establish that only a finite number of labels and a finite number of information trees have to be considered while modelchecking a formula with quantifiers.

Let $f n(S)=\bigcup_{A \in S} f n(A)$ for some $S \subseteq \mathcal{D}, f n(\rho)=\bigcup_{x \in \operatorname{dom}(\rho) \cap \mathcal{L}}\{\rho(x)\} \cup$ $\bigcup_{X \in \operatorname{dom}(\rho) \cap \tau} f n(\rho(X))$ for some valuation $\rho$ and $f n(\delta)=\bigcup_{\xi \in \operatorname{dom}(\delta)} f n(\delta(\xi))$ for some valuation $\delta$. For a formula $\varphi$ considered under some valuations $\rho$ and $\delta$, we define $f n(\varphi, \rho, \delta)$ as $f n(\varphi) \cup f n(\rho) \cup f n(\delta)$.

PROPOSITION 9 Let A be a tree and $\exists X . \varphi$ be a closed formula under some valuations $\rho$ and $\delta$. Let $n$ be a label which does not belong to $\mathrm{f}(A)$. Then $A F_{\rho, \delta} \exists X . \varphi$ iff there exists some tree $B$ in $\{C \mid C \sqsubseteq A\} \cup\{\{\{n[\{\}]\}\}\}$ such that $A \models_{\rho[X \rightarrow B], \delta} \varphi$. 
SKETCH OF PROOF. The right-to-left implication is an easy consequence of the definition of satisfaction. For the left-to-right implication: if $A \models \rho, \delta \exists X . \varphi$ then, by definition of satisfaction, there exists a tree $B$ such that $A \models_{\rho\left[X_{1}+B\right], \delta}$ $\varphi$. If this tree $B$ is a component of $A$, then the statement is obvious. Otherwise, we show that for any tree $C$ such that $C \nsubseteq A$, it holds that $A \models_{\rho(X \rightarrow C), \delta}$ iff

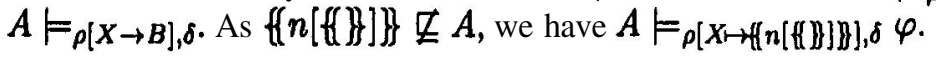

PROPOSITION 10 Let $A$ be a tree and $\exists x . \varphi$ be a closed formula under some valuations $\rho$ and $\delta$. Let $n$ be a label such that $n \notin f n(A) \cup f n(\varphi, \rho, \delta)$. Then $A \models_{\rho, \delta} \exists x . \varphi$ iff there exists some label $p$ in $f n(A) \cup f n(\varphi, \rho, \delta) \cup\{n\}$ such that $A=\rho[x \rightarrow p], \delta \varphi$.

SKETCH OF PROOF. The right-to-left direction is straightforward by the definition of satisfaction. For the left-to-right direction: as $A \models_{\rho, \delta} \exists x . \varphi$, by definition of satisfaction, there exists some label $p^{\prime}$ in $\Lambda$ such that $A \models_{\rho\left[p-p^{\prime}\right], \delta} \varphi$. If $p^{\prime} \in f n(A) \cup f n(\varphi, \rho, \delta)$ then the property obviously holds. Otherwise, we show that for any label $m$ such that $m \notin f n(A) \cup f n(\varphi, \rho, \delta)$, it holds that $A F_{\rho[n-m]}, \delta$ iff $A \models_{\rho\left[p-p^{\prime}\right], \delta} \varphi$. Therefore, $A F_{\rho[n-m], \delta} \varphi$.

\subsection{Polynomial Space Model-checking Algorithm}

We consider $\Delta$, a (possibly empty) sequence of the form $\left[\xi_{1} \rightarrow \varphi_{1}\right] \ldots\left[\xi_{n} \rightarrow\right.$ $\varphi_{n}$ ] where the $\varphi_{i}$ 's are formulas from the tree logic and $\xi_{i} \neq \xi_{j}$ whenever $i \neq j$. For all $i,\left[\xi_{i} \rightarrow \varphi_{i}\right]$ is the substitution which, when applied to some formula $\psi$, replaces the free occurrences of the recursion variable $\xi_{i}$ in $\psi$ by the formula $\varphi_{\boldsymbol{i}}$. The empty sequence is denoted $\epsilon$ and considered as the identity. The domain of a sequence of substitutions $\Delta$ (written $\operatorname{dom}(\Delta))$ is defined as (i) $\varnothing$ if $\Delta=\epsilon$ and $($ ii $)\left\{\xi_{1}, \ldots, \xi_{n}\right\}$ if $\Delta=\left[\xi_{1} \rightarrow \varphi_{1}\right] \ldots\left[\xi_{n} \rightarrow \varphi_{n}\right]$.

For a formula $\varphi, \Delta(\varphi)$ denotes the formula $\left[\xi_{1} \rightarrow \varphi_{1}\right] \circ \ldots \circ\left[\xi_{n} \rightarrow \varphi_{n}\right](\varphi)$. For a sequence of substitutions $\Delta, \Delta\langle\xi \leftarrow \varphi\rangle$ denotes the sequence of substitution $(i) \Delta[\xi \rightarrow \varphi]$ if $\xi \notin \operatorname{dom}(\Delta)$ and $(i i)\left[\xi_{1} \rightarrow \varphi_{1}\right] \ldots\left[\xi_{i} \rightarrow \varphi\right] \ldots\left[\xi_{n} \rightarrow\right.$ $\left.\varphi_{n}\right]$ if $\Delta=\left[\xi_{1} \rightarrow \varphi_{1}\right] \ldots\left[\xi_{n} \rightarrow \varphi_{n}\right]$ and $\xi=\xi_{i} \in \operatorname{dom}(\Delta)$.

We extend the mapping $f n$ to sequences of substitutions $\Delta: f n(\epsilon)=\varnothing$ and $f n(\Delta[\xi \rightarrow \varphi])=f n(\varphi) \cup f n(\Delta)$. For any formula $\varphi$, any valuation $\rho$ and any sequence of substitutions $\Delta$, we define $f n(\varphi, \rho, \Delta)=f n(\varphi) \cup f n(\rho) \cup f n(\Delta)$.

We present an algorithm $\operatorname{check}(A, \varphi, \rho, \Delta)$ with $A$ an information tree, $\varphi$ a tree logic formula, $\rho$ a valuation for label and tree variables and $\Delta$ a sequence of substitutions. This algorithm is correct in the sense of Theorem 11 below.

\section{Model-checking algorithm}

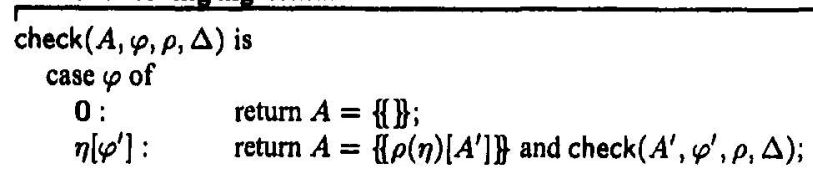




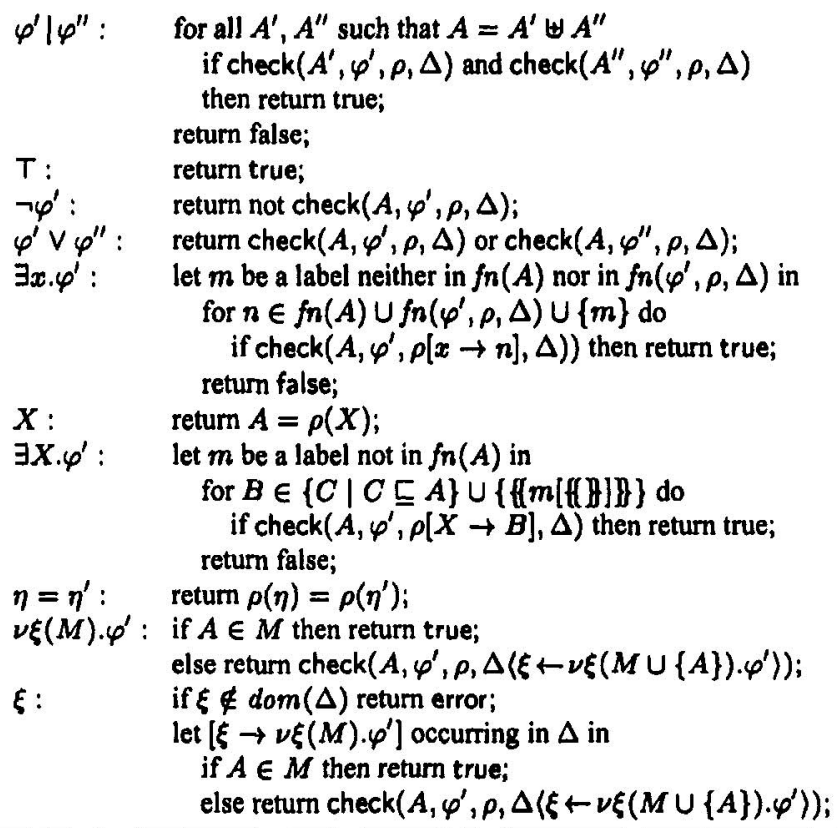

THEOREM 11 (CORRECTNESS) Let $\varphi$ be a $T L_{\nu}^{\exists}$ formula without free recursion variable and $\boldsymbol{\rho}$ be a valuation such that $\boldsymbol{\varphi}$ is $\rho$-closed, and let $A$ be an information tree. Then the evaluation of $\operatorname{check}(A, \varphi, \rho, \epsilon)$ terminates and computes true if $A \models \rho \varphi$ holds and false otherwise.

PROPOSITION 12 (COMPLEXITY) For any valuation $\boldsymbol{\rho}$, any $\boldsymbol{\rho}$-closed $T L_{\nu}^{\exists}$ formula $\varphi$ without free recursion variables and any information tree $A$, the evaluation of $\operatorname{check}(A, \varphi, \rho, \epsilon)$ requires polynomial space in the size of the model-checking problem $A \models_{\rho} \varphi$.

SKETCH OF PROOF. Any recursive call check $\left(A^{\prime}, \varphi^{\prime}, \rho^{\prime}, \Delta^{\prime}\right)$ generated by the evaluation of $\operatorname{check}(A, \varphi, \rho, \epsilon)$ has a polynomial-size representation. Moreover, the length of any chain of recursive calls of the algorithm check starting from check $(A, \varphi, \rho, \epsilon)$ is polynomially bounded. It is then sufficient to show that the evaluation of check $\left(A^{\prime}, \varphi^{\prime}, \rho^{\prime}, \Delta^{\prime}\right)$ can be done in polynomial space. For most of the cases of the "case" statement, it is obvious. The two non trivial cases are for $\varphi^{\prime}$ being $\psi^{\prime} \mid \psi^{\prime \prime}$ and $\exists X . \psi$. For the former, one has to consider all couples of trees $A^{\prime}, A^{\prime \prime}$ such that $A^{\prime} \uplus A^{\prime \prime}=A$. These couples correspond to all bipartitions of the multiset $A$ and so can be enumerated using linear space. For the latter, the set of all components of $A$ have to be considered. Remark that a component of $A$ corresponds to a subset of the set of nodes of $A$. This correspondence is not bijective, but one can determine in polynomial space whether 
a subset of the set of nodes of $A$ corresponds to a component of $A$. Therefore, the set of all components of $A$ can be enumerated in polynomial space. Note finally that all iterations of the evaluation loop reuse the same space.

As a consequence of Theorem 3, Proposition 12 and the inclusion $T L \subseteq$ $T L^{\exists} \subseteq T L_{\nu}^{\exists}$ we have

THEOREM 13 The model-checking problem for the tree logics TL, TL $L^{\exists}$ and $T L_{\nu}^{\exists}$ is PSPACE-complete.

\section{References}

[Abiteboul et al., 2000] Abiteboul, S., Buneman, P., and Suciu, D. (2000). Data on the Web. Morgan Kaufmann Publishers.

[Cardelli, 2000] Cardelli, L. (2000). Semistructured Computation. In 7th International Workshop on Database Programming Languages, DBPL'99, LNCS, pages 1-16. Springer.

[Cardelli and Ghelli, 2001] Cardelli, L. and Ghelli, G. (2001). A Query Language Based on the Ambient Logic. In European Symposium on Programming (ESOP'01), volume 2028 of $L N C S$, pages 1-22. Springer.

[Cardelli and Gordon, 2000a] Cardelli, L. and Gordon, A.D. (2000a). Anytime, Anywhere: Modal Logics for Mobile Ambients. In 27th ACM Symposium on Principles of Programming Languages (POPL'O0), pages 365-377.

[Cardelli and Gordon, 2000b] Cardelli, L. and Gordon, A.D. (2000b). Mobile Ambients. Theoretical Computer Science, 240:177-213.

[Charatonik et al., 2001] Charatonik, W., Dal Zilio, S., Gordon, A. D., Mukhopadhyay, S., and Talbot, J.-M. (2001). The Complexity of Model Checking Mobile Ambients. In Foundations of Software Science and Computation Structures (FoSSaCS'01), volume 2030 of LNCS, pages 152-167. Springer.

[Courcelle, 1990a] Courcelle, B. (1990a). Graph Rewriting: An Algebraic and Logic Approach. In Handbook of Theoretical Computer Science, volume B. Elsevier.

[Courcelle, 1990b] Courcelle, B. (1990b). The Monadic Second-Order Logic of Graphs. I. Recognizable Sets of Finite Graphs. Information and Computatution, 85(1): 12-75.

[Dal-Zilio and Lugiez, 2003] Dal-Zilio, S. and Lugiez, D. (2003). XML Schema, Tree Logic and Sheaves Automata. In Rewriting Techniques and Applications, 14th International Conference, RTA 2003, LNCS, pages 246-263. Springer.

[Neven, 2002] Neven, F. (2002). Automata, Logic and XML. In Annual Conference of the European Association for Computer Science Logic (CSL '02), volume 2471 of LNCS, pages 2-26. Springer.

[Seidl et al., 2003] Seidl, H., Schwentick, T., and Muscholl, A. (2003). Numerical Document Queries. In Twenty-Second ACM SIGACT-SIGMOD-SIGART Symposium on Principles of Database Systems, pages 155-166. ACM.

[Stirling and Walker, 1991] Stirling, C. and Walker, D. (1991). Local model checking in the modal mu-calculus. Theoretical Computer Science, 89:161-177.

[Stockmeyer, 1976] Stockmeyer, L. J. (1976). The Polynomial-time Hierarchy. Theoretical Computer Science, 3(1): 1-22.

[Winskel, 1991] Winskel, G. (1991). A note on model checking the modal $\nu$-calculus. Theoretical Computer Science, 83:157-167. 